\title{
A Novel Pilot Protection for VSC-HVDC Transmission Lines Based on Correlation Analysis*
}

\author{
Xingfu Jin , Guobing Song \\ School of Electrical Engineering, Xi'an Jiaotong University, Xi'an, Shaanxi, P. R. China \\ Email: xingfujin@stu.xjtu.edu.cn \\ Received February, 2013
}

\begin{abstract}
The control system of voltage source converter HVDC (VSC-HVDC) is complex and its fault tolerance ability is not sufficient, and correct rate of line protection device is not high. A novel pilot protection for VSC-HVDC transmission lines based on correlation analysis is proposed in this paper. In the principle, external fault is equivalent to a positive capacitance model, so the correlation coefficient of the current and voltage derivative is 1 ; while the internal fault is equivalent to a negative capacitance model, so the correlation coefficient of the current and voltage derivative is -1 . Internal faults and external faults can be distinguished by judging the correlation coefficient. Theoretical analysis and PSCAD simulation experiments show that the new principle, which is simple, not affected by transition resistance, control type and line distributed capacitance current, can identify internal faults and external faults reliably and rapidly, having certain practical value.
\end{abstract}

Keywords: VSC-HVDC; DC Transmission Lines; Correlation Coefficient; Pilot Protection

\section{Introduction}

Voltage Source Converter HVDC (VSC-HVDC) is a flexible and efficient DC transmission and distribution technology using full-controlled switching devices and high frequency PWM modulation technology, and it is very promising in the fields such as the grid connection of renewable energy, the island power supply, the urban power supply, the interconnection among synchronous grids and multi-terminal dc transmission [1-5]. Recently, ABB has successfully developed a HVDC circuit breaker [6], which provides the possibility for the formation of the DC grid.

Generally, the dc transmission lines are over long distance with a high fault rate, and need a set of perfect and reliable relay protection device to ensure the safe operation of the whole system. However, the relay protection of VSC-HVDC transmission line still adopt protection principle on CSC-HVDC lines which takes the traveling wave protection as the main protection and current differential protection as a backup protection, without taking into account the characteristics of VSC-HVDC [7-8]. The traveling wave protection is of fast response, not affected by $\mathrm{CT}$ saturation and long term distributed capacitance and so on, while it requires a high sampling

${ }^{*}$ This work was supported by the National Natural Science Foundation of China (No. 51177128) and the Key Program of National Natural Science Foundation of China (No. 51037005). frequency, is easily disturbed by the noise and loses efficacy with high resistance grounding. The current differential protection is effective for high resistance grounding, but is easily affected by distributed capacitance, requiring the long delay, which does not meet the fast control characteristics in dc transmission. In [9], a VSCHVDC cable lines differential protection principle is proposed based on frequency variable parameters model, reducing the effect of the distributed capacitance, but it increases a large amount of calculation and performs slowly with a low constant value and a long time delay to escape the transient process in order to ensure the unwanted operation in sound pole. In [10], a VSC-HVDC cable lines pilot direction protection principle is presented with RL model in circuit, but there is principle error and RLC in the model error function derive from a set of differential equations with a large amount of calculation [11]. Develop a pilot protection principle for VSC-HVDC cable lines using the current natural frequency. It also needs much calculation and the reliability depends on the accurate extraction of the natural frequency. Moreover, it needs extra auxiliary criterion and complex setting, and there exists the dead zone in both ends and the midpoint. In [12], a pilot protection method for the transmission lines is proposed on the basis of the principle of model identification. In this method, the internal faults are equivalent to the inductance models and the external faults are equivalent to the capacitance mod- 
els, so internal faults and external faults can be distinguished through calculating model error.

On the basis of the idea of model identification, this paper proposes a novel pilot protection principle for $\mathrm{dc}$ transmission lines combining with the characteristics of the VSC-HVDC whose double ends connect the shunt large capacitance. This new principle can accurately distinguish internal faults from external faults by judging the correlation coefficient of the current and the voltage derivative. It has the following characteristics:

- It is conducted in time dominant, needs a short data window, and has a fast response.

- The principle, simple and easily to be achieved, is not affected by the distributed capacitance.

- It has a strong patience to transition resistance, and can take action only on the fault pole.

\section{The Structure of VSC-HVDC and the Correlation Analysis}

\subsection{The Structure of VSC-HVDC}

Figure 1 is the simplified diagram of the structure of VSC-HVDC. $u_{\mathrm{Mp}}$ and $u_{\mathrm{Mn}}$ are respectively the measured voltage of the positive pole and the negative pole in M terminal. $i_{\mathrm{Mp}}$ and $i_{\mathrm{Mn}}$ are respectively the measured current of the positive pole and the negative pole in $\mathrm{M}$ terminal. $u_{\mathrm{Np}}$ and $u_{\mathrm{Nn}}$ are respectively the measured voltage of the positive pole and the negative pole in $\mathrm{N}$ terminal. $i_{\mathrm{Np}}$ and $i_{\mathrm{Nn}}$ are respectively the measured current of the positive pole and the negative pole in $\mathrm{N}$ terminal. $\mathrm{f}_{1}-\mathrm{f}_{5}$ are the fault location. $\mathrm{f}_{1}$ is inside the $\mathrm{dc}$ transmission line, $f_{2}$ and $f_{3}$ are outside the capacitors, $f_{4}$ and $f_{5}$ are in ac lines in front of primary side of the converter transformer in $\mathrm{M}$ terminal and $\mathrm{N}$ terminal. The reference directions of voltage and current are as shown in Figure 1.

The shunt large capacitance, on both sides of voltage source converter HVDC transmission lines, are mainly used to provide dc voltage for the converter, and at the same time, they can buffer the fluctuation in dc side when the fault occurs, reduce the voltage ripple of the dc side and support dc voltage of the receiving end [2].

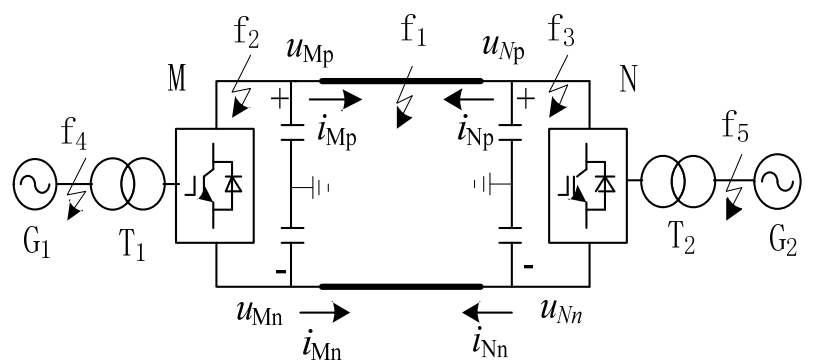

Figure 1. VSC-HVDC transmission system with two electrodes.

\subsection{The Correlation Analysis}

Correlation analysis is used to describe the relevance of two variables or more. Linear correlation analysis is used to represent the linear correlation degree of two variables, and usually employs the correlation coefficient as a numerical index. From [13], it can be noted that the correlation coefficient between two variables can be expressed as follows:

$$
\rho_{x y}=\frac{\int_{-\infty}^{\infty} x(t) y(t) d t}{\sqrt{\int_{-\infty}^{\infty} x^{2}(t) d t} \sqrt{\int_{-\infty}^{\infty} y^{2}(t) d t}}
$$

After discretization:

$$
\rho_{x y}=\frac{\sum_{k=1}^{N} x(k) y(k)}{\sqrt{\sum_{k=1}^{N} x^{2}(k) \sum_{k=1}^{N} y^{2}(k)}}
$$

The correlation coefficient $\rho$ is a dimensionless value, varying from -1 to 1 . If $\rho$ is 1 , it stands for positive linear relationship between variables, while if $\rho$ is -1 , it represents negative linear relationship between variables. If $\rho$ is 0 , it stands that the two variables have no relation at all.

\section{The Analysis of the Fault Characteristics of VSC-HVDC Transmission Lines}

\subsection{Model of the External Fault}

On the basis of superposition theorem, the dc lines shown in Figure 1 can be equivalent to the superposition of a circuit network in normal operation and a fault network [14]. Setting the external fault in M end as an example, its fault components network is shown in Figure 2 , where $\mathrm{u}_{\mathrm{f}}$ is the voltage source of fault components network, $\mathrm{C}_{\mathrm{p}}$ is the shunt large capacitance of the line ends; the lines adapt $\pi$ model, and $\mathrm{L}, \mathrm{R}$ and $\mathrm{C}$ are the corresponding equivalent inductance, resistance and capacitance with the lumped parameter; the inductive converters can be equivalent to the $R_{\text {sn }}$ resistance and the $L_{s n}$ inductance [2].

In this paper, we suppose that the positive direction of current is from the converter to the line. From the basic circuit principle, we can obtain the following equations:

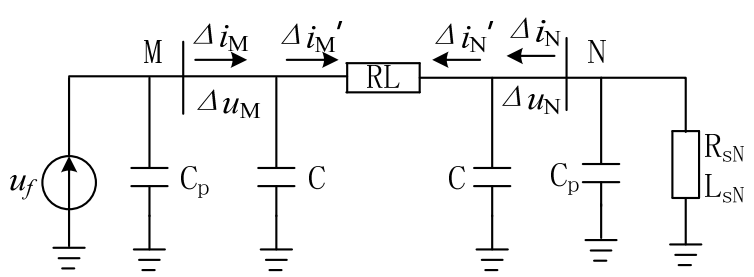

Figure 2. The fault components net of external faults. 


$$
\left\{\begin{array}{l}
\Delta i_{\mathrm{M}}{ }^{\prime}=\Delta i_{\mathrm{M}}-\mathrm{C} \frac{\mathrm{d} \Delta u_{\mathrm{M}}}{\mathrm{d} t} \\
\Delta i_{\mathrm{N}}{ }^{\prime}=\Delta i_{\mathrm{N}}-\mathrm{C} \frac{\mathrm{d} \Delta u_{\mathrm{N}}}{\mathrm{d} t} \\
\Delta i_{\mathrm{M}}{ }^{\prime}+\Delta i_{\mathrm{N}}{ }^{\prime}=0
\end{array}\right.
$$

The fault components differential current and differential voltage is defined as follows:

$$
\left\{\begin{array}{l}
\Delta i_{\mathrm{cd}}=\Delta i_{\mathrm{M}}+\Delta i_{\mathrm{N}} \\
\Delta u_{\mathrm{cd}}=\Delta u_{\mathrm{M}}+\Delta u_{\mathrm{N}}
\end{array}\right.
$$

Then we can obtain the following equation with the external fault:

$$
\Delta i_{\mathrm{cd}}=\mathrm{C} \frac{\mathrm{d} \Delta u_{\mathrm{cd}}}{\mathrm{d} t}
$$

From the analysis of the Equation (5) we can achieve the following equivalent positive capacitance model of the external fault, as shown in Figure 3

From the Equation (5) we can obtain the correlation coefficient of the differential current and differential voltage derivative as follows:

$$
\rho\left(\Delta i_{\mathrm{cd}}, \frac{\mathrm{d} \Delta u_{\mathrm{cd}}}{\mathrm{d} t}\right)=1
$$

\subsection{The Model of the Internal Fault}

The fault components network is shown in Figure 4 when the internal fault occurs in dc transmission lines. $R_{s M}, L_{s M}, R_{s N}$ and $L_{s N}$ are respectively the equivalent resistance and inductance of the $\mathrm{M}$ and $\mathrm{N}$ terminal.

At the moment of a sudden fault, the shunt large capacitance will rapidly discharge to the fault point, producing a huge impulse current. As shown in Figure 4, the impulse current $\Delta i_{\mathrm{cM}}$ and $\Delta i_{\mathrm{cN}}$ are respectively far larger than $\Delta i_{\mathrm{sM}}$ and $\Delta i_{\mathrm{sN}}$, and the system side can be equivalent to the shunt large capacitance [9-11].

From Figure 4, we can obtain the following equations based on the basic circuit principle with $\Delta i_{s \mathrm{M}}$ and $\Delta i_{\mathrm{sN}}$ neglected.

$$
\left\{\begin{array}{l}
-\Delta i_{\mathrm{M}}=\Delta i_{\mathrm{cM}}=\mathrm{C}_{\mathrm{p}} \frac{\mathrm{d} \Delta u_{\mathrm{M}}}{\mathrm{d} t} \\
-\Delta i_{\mathrm{N}}=\Delta i_{\mathrm{cN}}=\mathrm{C}_{\mathrm{p}} \frac{\mathrm{d} \Delta u_{\mathrm{N}}}{\mathrm{d} t}
\end{array}\right.
$$

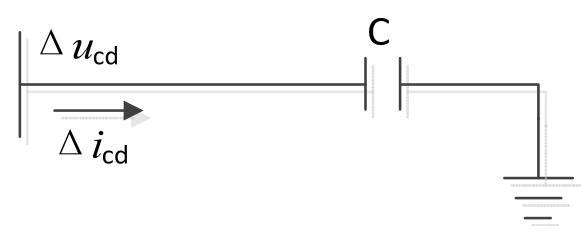

Figure 3. Positive capacitance model of external faults.
According to the definition of the differential current and voltage, the following equation is made as:

$$
-\Delta i_{\mathrm{cd}}=\mathrm{C}_{\mathrm{p}} \frac{\mathrm{d} \Delta u_{\mathrm{cd}}}{\mathrm{d} t}
$$

From the analysis of the Equation (8), we can achieve the following equivalent negative capacitance model of internal faults, as shown in Figure 5.

From the Equation (8) we can obtain the correlation coefficient of the differential current and differential voltage derivative as follows:

$$
\rho\left(\Delta i_{\mathrm{cd}}, \frac{\mathrm{d} \Delta u_{\mathrm{cd}}}{\mathrm{d} t}\right)=-1
$$

\section{Principle and Criterion of the Pilot Protection}

On the basis of part III, the correlation coefficient of the current and voltage derivative is 1 when an external fault occurs; while the correlation coefficient of the current and voltage derivative is -1 when an internal fault occurs. Then we can make the criterion of the pilot protection based on this characteristic.

The criterion by comparing correlation coefficient is as follows:

$$
\rho\left(\Delta i_{\mathrm{cd}}, \frac{\mathrm{d} \Delta u_{\mathrm{cd}}}{\mathrm{d} t}\right)<\rho_{\text {set }}
$$

where $\rho_{\text {set }}$ is the threshold, usually assuming from -0.8 to 0 , and the length of the data window is $5 \mathrm{~ms}$.

The specific process of this algorithm is as follows: firstly, we can use the difference algorithm to calculate the voltage and current fault components; then extract low frequency components through filtering in low pass band and calculate the correlation coefficient of the differential current and differential voltage derivative with the least squares algorithm; lastly, we can distinguish the faults by comparing the calculated value with the set value.

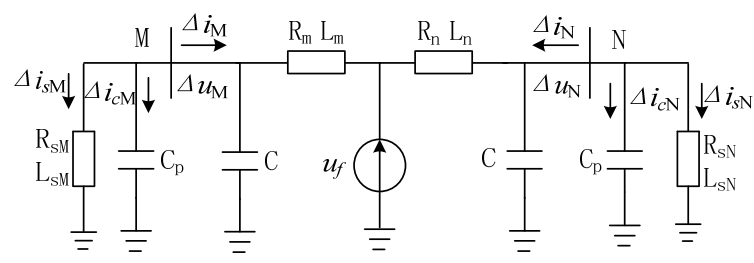

Figure 4. The fault components network of internal faults.

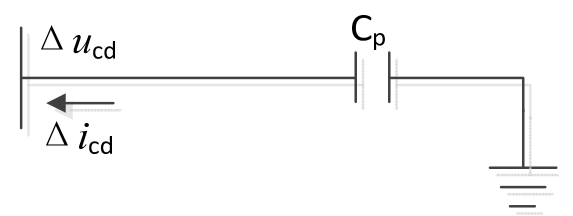

Figure 5. Negative capacitance model of internal line faults. 
It is necessary to make instructions that lines in this paper adopt $\pi$ model equivalent circuit corresponding to a certain applicable band, so the method needs low pass filtering [15].

\section{Simulations}

The $\pm 60 \mathrm{kV}$ bipolar VSC-HVDC transmission system simulation models are shown in Figure 1. The system capacity is $60 \mathrm{MW}$, and the line length is $300 \mathrm{~km}$. PSCAD is employed for electromagnetic transient simulation and MATLAB is used for data processing.

In this simulation model, the frequency-dependent parameters line model is used, and its structure and control strategy is detailed in [9]. The shunt capacitances of both the positive and negative pole are $1000 \mathrm{~F}$ and the sampling rate is $10 \mathrm{kHz}$. The fault occurs at $2.5 \mathrm{~s}$ with duration of $0.1 \mathrm{~s}$ in the system. The data window is about 5 $\mathrm{ms}$ and the action threshold $\rho_{\text {set }}$ is set to be -0.7 . Because of the limited space, this paper only presents the fault simulation results under the condition of the most unfavorable to the protection action.

\subsection{The Simulation Results of Internal Faults}

The simulation results are shown in Figure 6 when an internal fault occurs at the $30 \mathrm{~km}$ point from the terminal $\mathrm{M}$ with a transition resistance of 300 in the positive pole. The Figure 7 presents the simulation results when a metallic grounding fault occurs at the $270 \mathrm{~km}$ point from the terminal $\mathrm{M}$ in the positive pole.

From Figures 6 and 7, it can be noted that when a fault with different fault distance and different transition resistance occurs, for the fault pole, the correlation coefficient $\rho$ is far less than the threshold $\rho_{\text {set }}$ and protection devices can accurately distinguish fault; for the sound pole, it is equivalent to the external faults, and the correlation coefficient $\rho$ is far more than the threshold $\rho_{\text {set }}$, which can ensure the protection do not

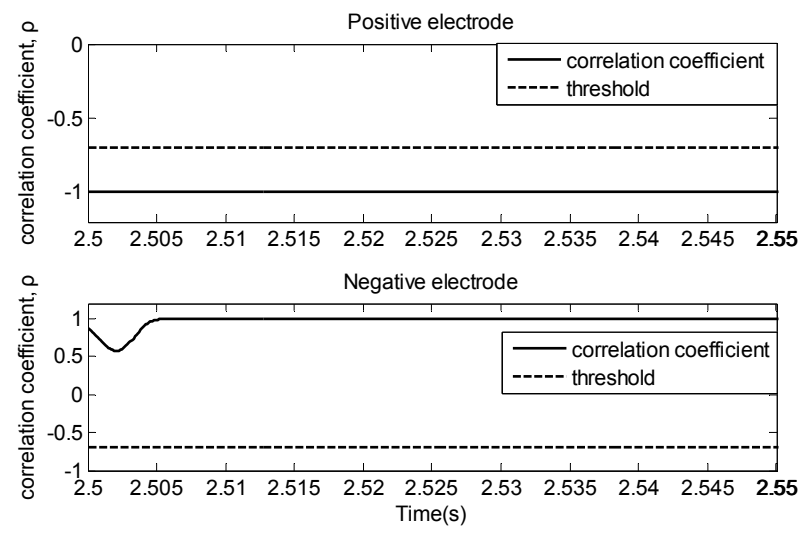

Figure 6. Simulation results when a fault at $30 \mathrm{~km}$ from the M-side with $300 \Omega$ resistances occurs.
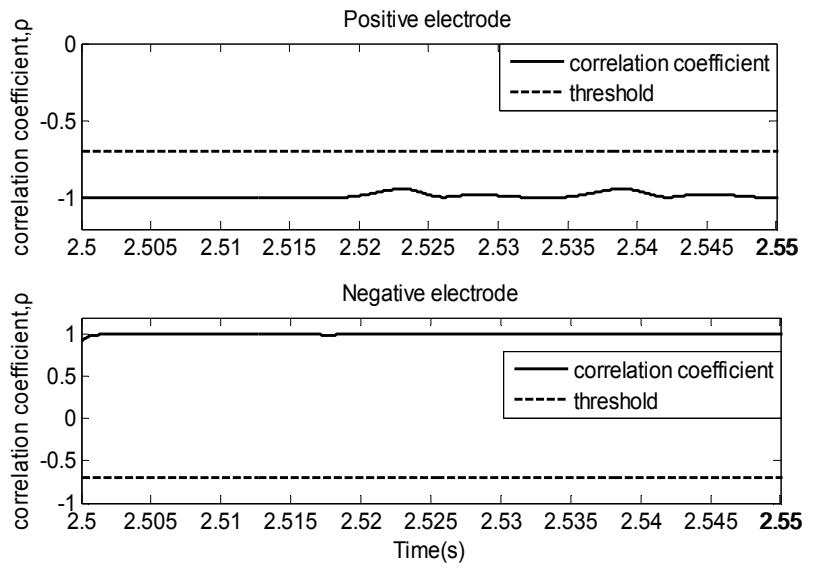

Figure 7. Simulation results when a metallic ground fault at $30 \mathrm{~km}$ from the M-side occurs.

take action.

\subsection{The Simulation Results of External Faults}

Simulation results when a metallic ground fault in DC lines beyond the M-side(corresponding to $f_{2}$ in Figure 1) occurs are shown in Figure 8; Figure 9 presents the simulation results when a metallic ground fault in $\mathrm{AC}$ lines beyond the $\mathrm{N}$-side(corresponding to $\mathrm{f}_{5}$ in Figure 1) occurs.

From Figure 8 and Figure 9, it can be seen that the correlation coefficient $\rho$ is far more than the threshold $\rho_{\text {set }}$ when the external fault occurs with different fault distance and different transition resistance, which can ensure the protection do not take action.

A lot of simulation results show that the protections can correctly take action when internal faults in dc line occur, and can reliably ensure inaction when external faults in dc line occur.

\section{Conclusions}

This paper proposes a novel pilot protection principle for VSC-HVDC transmission lines based on correlation analysis. Theoretical analysis and simulation results can help draw the following conclusions:

- The positive capacitance model reflects the external faults and negative capacitance model reflects the internal faults. Comparing the correlation coefficient of differential current and differential voltage derivative with the threshold, we can rapidly and reliably distinguish internal faults from external faults.

- The faults for the fault pole are internal, but external for the sound pole, and the correlation coefficient of differential current and differential voltage derivative is 1 . Therefore this protection can take action only on the fault pole. 


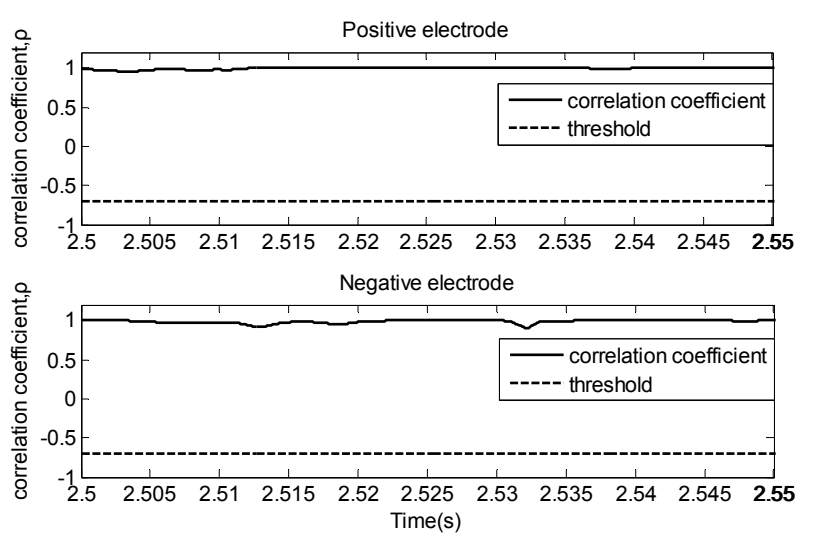

Figure 8. Simulation results when a metallic ground fault in DC lines beyond the M-side occurs.
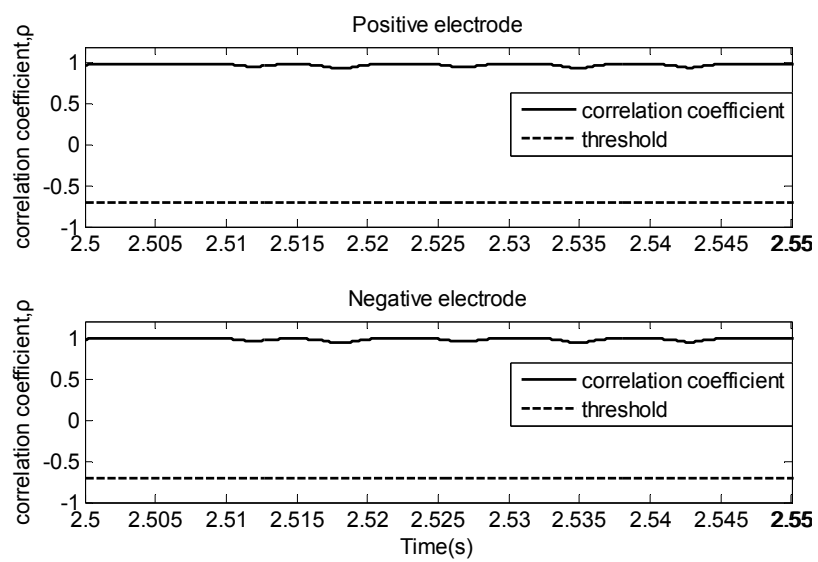

Figure 9. Simulation results when a metallic ground fault in AC lines beyond the $\mathrm{N}$-side occurs.

- The proposed novel pilot protection principle regards the correlation coefficient as criteria without compensating capacitor current. This principle is simple and easy to be achieved. Simulation results indicate that this principle is not affected by transition resistance, control type and it can identify internal faults and external faults reliably and rapidly under various working conditions, having certain practical value.

\section{REFERENCES}

[1] "HVDC \& FACTS Group of Zhejiang University Power Generating Department, "DC transmission," Beijing: Electric Power Industry Press, 1985, pp. 1-24.

[2] G. F. Tang, "HVDC Transmission Technology Based
Voltage Source Converter," Beijing: China Electric Power Press, 2010, pp. 2-36.

[3] Z. Xu and H. R. Chen, "Review and Applications of VSC HVDC," High Voltage Engineering, Vol. 30, No. 1, 2007, pp. 1-10.

[4] Z. Q. Hu, C. X. Mao, J. M. Lu, et al., "New High Voltage Direct Current Transmission Technology_ HVDC light," Transactions of Chinese Electrotechnical Society, Vol. 20, No. 7, 2005, pp. 12-16.

[5] G. Y. Li, P. F. Lv, G. K. Li, et al., "Development and Prospects for HVDC Light," Automation of Electric Power Systems, Vol. 27, No. 4, 2003, pp. 77-80.

[6] ABB, "ABB Solves 100-year-old Electrical Puzzle - New Technology to Enable Future DCgrid [eb/ol]," [2012-11-7].http://www.abb.com.cn/cawp/seitp202/8c6c0 7b41d96890648257ab0001f5ac3.aspx.

[7] M. X. Han and J. Wen, "The Principle and Operation of HVDC," Beijing: CHINA MACHINE PRESS, 2009, pp. 185-205.

[8] ABB, "It's Time to Connect-technical Description of HVDC Light Technology," Sweden: ABB Power Technology, 2005.

[9] G. B. Song, X. L. Cai, S. P. Gao, et al., "A New Current Differential Protection Principle of VSC-HVDC Considering Frequency-dependent Characteristic of Cable Line," Proceedings of the CSEE, Vol. 31, No. 22, 2011, pp. 105-111.

[10] G. B. Song, X. L. Cai, S. P. Gao, et al., "Directional Element for VSC-HVDC Transmission Lines Based on Model Identification," Power System Protection and Control, Vol. 40, No. 7, 2012, pp. 78-83.

[11] G. B. Song, X. L. Cai, S. P. Gao, et al., "New Pilot Protection for VSC-HVDC Transmission Lines Based on Natural Frequency Characteristic of Current," High Voltage Engineering, Vol. 37, No. 8, 2011, pp. 1989-1996.

[12] J. Suonan, C. Yang, Z. L. Yang, et al., "New Type of Transmission Line Pilot Protection Based on Model Identification," Automation of Electric Power Systems, Vol. 32, No. 24, 2008, pp. 30-34.

[13] S. Li, G. B. Song, X. N. Kang, et al., "Time-domain Fault Line Selection Based on Correlation Analysis in Neutral Indirect Ground System," Power System Protection and Control, Vol. 36, No. 13, 2008, pp. 16-20.

[14] Y. Z. Ge, "Principle and Technology of New Relay Protection and Fault Location," Version 2. Xi'an: Xi'an Jiaotong University Press, 2007.

[15] X. N. Kang, Z. Z. Wu, J. Suonan, et al., "Research of Suitable Frequency Band of Transmission Line Model Applied to Pilot Differential Protection Based on Parameter Identification," Power System Protection and Control, Vol. 39, No. 19, 2011, pp. 8-16. 